\title{
Convergence and Stability of the Split-Step $\theta$-Milstein Method for Stochastic Delay Hopfield Neural Networks
}

\author{
Qian Guo, ${ }^{1,2}$ Wenwen Xie, ${ }^{1}$ and Taketomo Mitsui ${ }^{3}$ \\ ${ }^{1}$ Department of Mathematics, Shanghai Normal University, Shanghai 200234, China \\ ${ }^{2}$ Division of Computational Science, E-Institute of Shanghai Universities, 100 Guilin Road, Shanghai 200234, China \\ ${ }^{3}$ Department of Mathematical Sciences, Faculty of Science and Engineering, Doshisha University, Kyoto 610-0394, Japan
}

Correspondence should be addressed to Qian Guo; qguo@shnu.edu.cn

Received 8 December 2012; Accepted 26 February 2013

Academic Editor: Chengming Huang

Copyright (C) 2013 Qian Guo et al. This is an open access article distributed under the Creative Commons Attribution License, which permits unrestricted use, distribution, and reproduction in any medium, provided the original work is properly cited.

\begin{abstract}
A new splitting method designed for the numerical solutions of stochastic delay Hopfield neural networks is introduced and analysed. Under Lipschitz and linear growth conditions, this split-step $\theta$-Milstein method is proved to have a strong convergence of order 1 in mean-square sense, which is higher than that of existing split-step $\theta$-method. Further, mean-square stability of the proposed method is investigated. Numerical experiments and comparisons with existing methods illustrate the computational efficiency of our method.
\end{abstract}

\section{Introduction}

Hopfield neural networks, which originated with Hopfield in the 1980s [1], have been successfully applied in many areas such as combinatorial optimization [2,3], signal processing [4], and pattern recognition $[5,6]$. In the last decade, neural networks in the presence of signal transmission delay and stochastic perturbations, also named as stochastic delay Hopfield neural networks (SDHNNs), have gained considerable research interest (see, e.g., [7-9] and the references therein). It is noticed that, so far, most works on SDHNNs focus mainly on the stability analysis of the analytical solutions, including mean-square exponential stability [7], global asymptotic stability [9], and so forth. Not only simulation is an important tool to explore interesting dynamics of kinds of Hopfield neural networks (HNNs) (see, e.g., [10] and the references therein), but also parameter estimation in dynamical systems based on HNNs (see, e.g., [11]) needs to solve HNNs numerically. Moreover, because most of SDHNNs do not have explicit solutions, the numerical analysis of SDHNNs recently stirred some initial research attention. For example, Li et al. [12] investigated the exponential stability of the Euler method and the semi-implicit Euler method for SDHNNs.
Rathinasamy [13] introduced a split-step $\theta$-method (SST) for SDHNNs and analysed the mean-square stability of this method, and the SST is only given for the commensurable delay case. To the best of our current knowledge, the authors mainly discussed the stability of numerical solutions for stochastic Hopfield neural networks with discrete time delays but skipped the details of convergence analysis.

The split-step Euler method for stochastic differential equations (SDEs) was proposed by Higham et al. [14], further, the splitting Euler-type algorithms have been derived for stochastic delay differential equations (SDDEs) $[15,16]$. In this paper, we will present a splitting method with higher order convergence for SDHNNs. To be specific, we will go into detail about the convergence analysis and comparing the stability with split-step $\theta$-method given in [13].

The rest of this paper is organized as follows. In Section 2, we recall the stochastic delay neural networks model and present a split-step $\theta$-Milstein method. In Section 3, we derive the convergence results of the split-step $\theta$-Milstein method for the model. In Section 4, the numerical stability analysis is performed. In Section 5, some numerical examples are given to confirm the theory. In the last Section, we draw some conclusions. 


\section{Model and the Split-Step $\boldsymbol{\theta}$-Milstein Method}

2.1. Model. Consider the stochastic delay Hopfield neural networks of the form

$$
\begin{aligned}
\mathrm{d} \mathbf{x}(t)= & {[-C \mathbf{x}(t)+A \mathbf{f}(\mathbf{x}(t))+B \mathbf{g}(\mathbf{z}(t))] \mathrm{dt} } \\
& +\Phi(\mathbf{x}(t)) \mathrm{d} W(t),
\end{aligned}
$$

where $\mathbf{x}(t)=\left[x_{1}(t), \ldots, x_{n}(t)\right]^{T} \in \mathbb{R}^{n}$ is the state vector associated with the $n$ neurons, $\mathbf{z}(t)=\left[x_{1}\left(t-\tau_{1}\right), \ldots, x_{n}\left(t-\tau_{n}\right)\right]^{T}$, the diagonal matrix $C=\operatorname{diag}\left(c_{1}, c_{2}, \ldots, c_{n}\right)$ has positive entries, and $c_{i}$ represents the rate at which the ith unit will reset its potential to the resting state in isolation when discounted from the network and the external stochastic perturbation. The matrices $A=\left(a_{i j}\right)_{n \times n}$ and $B=\left(b_{i j}\right)_{n \times n}$ are the connection weight matrix and the discretely delayed connection weight matrix, respectively. Furthermore, the vector functions $\mathbf{f}(\mathbf{x}(t))=\left[f_{1}\left(x_{1}(t)\right), \ldots, f_{n}\left(x_{n}(t)\right)\right]^{T}$ and $\mathbf{g}(\mathbf{z}(t))=\left[g_{1}\left(x_{1}\left(t-\tau_{1}\right)\right), \ldots, g_{n}\left(x_{n}\left(t-\tau_{n}\right)\right)\right]^{T}$ denote the neuron activation functions with the conditions $f_{i}(0)=$ $0, g_{i}(0)=0$ for all positive $\tau_{j}$.

On the initial segment $[-\tau, 0]$ the state vector satisfies $\mathbf{x}(t)=\psi(t)$, where $\psi(t)=\left[\psi_{1}(t), \ldots, \psi_{n}(t)\right]^{T}$ is a given function in $C\left([-\tau, 0], \mathbb{R}^{n}\right)$ and $\tau$ stands for $\max _{1 \leq i \leq n}\left\{\tau_{i}\right\}$.

Moreover, $\Phi(\mathbf{x}(t))=\operatorname{diag}\left(\phi_{1}\left(x_{1}(t)\right), \ldots, \phi_{n}\left(x_{n}(t)\right)\right)$ is a diagonal matrix with $\phi_{i}(0)=0$ and $W(t)=\left[W_{1}(t), \ldots\right.$, $\left.W_{n}(t)\right]^{T} \in \mathbb{R}^{n}$ is an $n$-dimensional Wiener process defined on the complete probability space $\left(\Omega, \mathscr{F},\left\{\mathscr{F}_{t}\right\}_{t \geq 0}, \mathbb{P}\right)$ with a filtration $\left\{\mathscr{F}_{t}\right\}_{t \geq 0}$ satisfying the usual conditions (i.e., it is increasing and right continuous while $\mathscr{F}_{0}$ contains all $\mathbb{P}$-null sets).

Let $f_{i}$ and $g_{i}$ be functions in $C^{2}(D ; \mathbb{R}) \bigcap \mathscr{L}^{2}([0, T] ; \mathbb{R})$ and $\phi_{i}$ be in $C^{1}(D ; \mathbb{R}) \cap \mathscr{L}^{2}([0, T] ; \mathbb{R})$. Here $C^{l}(D ; \mathbb{R})$ denotes the family of continuously $l$-times differentiable real-valued function defined on $D$, while $\mathscr{L}^{l}([0, T] ; \mathbb{R})$ denotes the family of all real-valued measurable $\left\{\mathscr{F}_{t}\right\}$-adapted stochastic processes $\{f(t)\}_{t \in[0, T]}$ such that $\int_{0}^{T}|f(t)|^{l} d t<+\infty$.

2.2. Numerical Scheme. We define the mesh with a uniform step-size $h(0<h<1)$ on the interval $[0, T]$; that is, $t_{k}=$ $k \cdot h(k=0,1, \ldots, K)$ and $T=K h$.

Let $\Delta W_{i}^{k}=W_{i}\left(t_{k+1}\right)-W_{i}\left(t_{k}\right)$ denote the increment of the Wiener process. The split-step $\theta$-Milstein (SSTM) scheme for the solution of SDEs (1) is given by

$$
\begin{aligned}
& Y^{k}=X^{k}+\left[-(1-\theta) C X^{k}-\theta C Y^{k}+A \mathbf{f}\left(X^{k}\right)+B \mathbf{g}\left(Z^{k}\right)\right] h, \\
& X^{k+1}=Y^{k}+\Phi\left(Y^{k}\right) \Delta W^{k}+\frac{1}{2} \widehat{\Phi}\left(Y^{k}\right)\left[\Delta W^{k} \circ \Delta W^{k}-1 h\right],
\end{aligned}
$$

where the merging parameter $\theta$ satisfies $0 \leq \theta \leq 1, X^{k}=$ $\left[X_{1}^{\mathrm{k}}, \ldots, X_{n}^{k}\right]^{T}$ is an approximation to $\mathbf{x}\left(t_{k}\right)$, and for $1 \leq q_{j} \in$ $\mathbb{Z}^{+}$

$$
Z_{j}^{k}= \begin{cases}\psi_{j}\left(t_{k}-\tau_{j}\right), & t_{k}-\tau_{j} \leq 0 \\ X_{j}^{k-q_{j}}, & 0<t_{k}-\tau_{j} \in\left[t_{k-q_{j}}, t_{k-q_{j}+1}\right) .\end{cases}
$$

Moreover, we adopt the symbols $\widehat{\Phi}\left(Y^{k}\right)=$ $\operatorname{diag}\left(\phi_{1}^{\prime}\left(Y_{1}^{k}\right) \phi_{1}\left(Y_{1}^{k}\right), \ldots, \phi_{n}^{\prime}\left(Y_{n}^{k}\right) \phi_{n}\left(Y_{n}^{k}\right)\right)$ and $\Delta W^{k}=\left[\Delta W_{1}^{k}\right.$, $\left.\ldots, \Delta W_{n}^{k}\right]^{T}$, the Hadamard product $\Delta W^{k} \circ \Delta W^{k}$ means $\left[\left(\Delta W_{1}^{k}\right)^{2}, \ldots,\left(\Delta W_{n}^{k}\right)^{2}\right]^{T}$, and $\mathbb{1}=[1, \ldots, 1]^{T} \in \mathbb{R}^{n}$. When $t_{k} \leq 0$, we define $X^{k}=\psi\left(t_{k}\right)$.

Then scheme (2) can be written in equivalent form as

$$
\begin{aligned}
& Y^{k}=X^{k}-(I+\theta C h)^{-1} C h X^{k} \\
& +(I+\theta C h)^{-1} h\left[A \mathbf{f}\left(X^{k}\right)+B \mathbf{g}\left(Z^{k}\right)\right], \\
& X^{k+1}=Y^{k}+\Phi\left(Y^{k}\right) \Delta W^{k}+\frac{1}{2} \widehat{\Phi}\left(Y^{k}\right)\left[\Delta W^{k} \circ \Delta W^{k}-\mathbb{1} h\right] \text {. }
\end{aligned}
$$

Substituting (4a) into (4b), we have a stochastic explicit single-step method with an increment function $\Lambda(\xi, \eta$, $\left.h, \Delta W^{k}\right) \in \mathbb{R}^{n}$; that is,

$$
X^{k+1}=X^{k}+\Lambda\left(X^{k}, Z^{k}, h, \Delta W^{k}\right) .
$$

\section{Order and Convergence Results for SSTM}

In this section we consider the global error of SSTM (2) as applied to SDHNNs (1) with initial condition. In what follows, $\|\cdot\|$ denotes Euclidean norm in $\mathbb{R}^{n}$.

For convergence purpose we make the following standard assumptions.

Assumption 1. Assume that $\mathbf{f}, \mathbf{g}, \boldsymbol{\Phi}$, and $\widehat{\boldsymbol{\Phi}}$ satisfy the Lipschitz condition

$$
\begin{aligned}
& \qquad \begin{array}{l}
\left|f_{i}(a)-f_{i}(b)\right| \leq \alpha_{i}|a-b|, \\
\left|g_{i}(a)-g_{i}(b)\right| \leq \beta_{i}|a-b|, \\
\left|\phi_{\mathrm{i}}(a)-\phi_{i}(b)\right| \leq \gamma_{i}|a-b|, \\
\left|\phi_{i} \phi_{i}^{\prime}(a)-\phi_{i} \phi_{i}^{\prime}(b)\right| \leq \kappa_{i}|a-b|, \\
\text { for every } i \text { and the linear growth condition } t \in \\
\qquad \mathbf{f}(\mathbf{x})\left\|^{2} \vee\right\| \mathbf{g}(\mathbf{x})\left\|^{2} \vee\right\| \Phi(\mathbf{x}) \cdot \mathbb{1}\left\|^{2} \vee\right\| \widehat{\Phi}(\mathbf{x}) \cdot \mathbb{1} \|^{2} \\
\leq \widetilde{L}\left(1+\|\mathbf{x}\|^{2}\right), \quad \forall \mathbf{x} \in \mathbb{R}^{n},
\end{array}
\end{aligned}
$$$$
(a, b \in \mathbb{R} \text { and } t \in[0, T])
$$

where $\widetilde{L}$ is a positive constant and $\vee$ is the maximal operator. We also define $L_{i}$ as $L_{i}=\max \left\{\alpha_{i}, \beta_{i}, \gamma_{i}, \kappa_{i}\right\}(i=1,2, \ldots, n)$.

We also need the following assumption on the initial condition.

Assumption 2. Assume that the initial function $\psi(t)$ is Lipschitz continuous from $[-\tau, 0]$ to $\mathbb{R}^{n}$, that is, there is a positive constant $L_{\psi}$ satisfying

$$
\|\psi(t)-\psi(s)\| \leq L_{\psi}(t-s) \quad \text { if }-\tau \leq s<t<0 .
$$


Now we give the definition of local and global errors.

Definition 1. Let $\mathbf{x}(t)$ denote the exact solution of (1). The local approximate solution $\widetilde{\mathbf{x}}\left(t_{k+1}\right)$ starting from $\mathbf{x}\left(t_{k}\right)$ by SSTM (2) given by

$$
\widetilde{\mathbf{x}}\left(t_{k+1}\right):=\mathbf{x}\left(t_{k}\right)+\Lambda\left(\mathbf{x}\left(t_{k}\right), \widetilde{Z}\left(t_{k}\right), h, \Delta W^{k}\right),
$$

where $\widetilde{Z}\left(t_{k}\right)$ denotes the evaluation of (3) using the exact solution, yields the difference

$$
\delta^{k+1}:=\mathbf{x}\left(t_{k+1}\right)-\widetilde{\mathbf{x}}\left(t_{k+1}\right)
$$

Then the local error of SSTM is defined by $\left\|\delta^{k+1}\right\|$, whereas its global error means $\left\|\epsilon^{k}\right\|$ where $\epsilon^{k}:=\mathbf{x}\left(t_{k}\right)-X^{k}$.

Definition 2. If the global error satisfies

$$
E\left(\left\|\epsilon^{k}\right\|\right)^{2} \leq \Gamma h^{2 p} \quad \forall h \in\left(0, h_{0}\right)
$$

with positive constants $h_{0}$ and $\Gamma$ and a finite $p$, then we say that the order of mean-square convergence accuracy of the method is $p$. Here $E$ is the expectation with respect to $\mathbb{P}$.

We then give the following lemmas that are useful in deriving the convergence results.

Lemma 3 (see also [17]). Let the linear growth condition (7) hold, and the initial function $\psi(t)$ is assumed to be $\mathscr{F}_{0}$-measurable and right continuous. And one puts $E_{\psi}:=$ $E\left(\sup _{-\tau \leq t \leq 0}\|\psi(t)\|^{2}\right)<\infty$. For any given positive $T$, there exist positive numbers $\Gamma_{\psi}$ and $\Gamma_{2}$ such that the solution of (1) satisfies

$$
E\left(\sup _{-\tau \leq s \leq T}\|\mathbf{x}(s)\|^{2}\right) \leq \Gamma_{\psi}
$$

where the constant $\Gamma_{\psi}$ is independent of step-size $h$ but dependent on T. Moreover, for any $0 \leq s<t \leq T, t-s<1$, the estimation

$$
E(\|\mathbf{x}(t)-\mathbf{x}(s)\|)^{2} \leq \Gamma_{2}(t-s)
$$

holds.

The Jensen inequality derives

$$
E\left(\sup _{-\tau \leq s \leq T}\|\mathbf{x}(s)\|\right) \leq \sqrt{\Gamma_{\psi}}
$$

from (12).

Lemma 4. For $s \in\left[t_{k}, t_{k}+h\right]$, one has

$$
E\left(\left\|\mathbf{z}(s)-\widetilde{Z}\left(t_{k}\right)\right\|^{2}\right) \leq \Gamma_{\tau} h .
$$

Here the constant $\Gamma_{\tau}$ is independent of step-size $h$.
Proof. If $t_{k}-\tau_{j} \leq 0$ and $s-\tau_{j} \leq 0$, under Assumption 2 we have

$$
\begin{aligned}
& E\left[x_{j}\left(s-\tau_{j}\right)-\widetilde{Z}_{j}\left(t_{k}\right)\right]^{2} \\
& \quad=E\left[\psi_{j}\left(s-\tau_{j}\right)-\psi_{j}\left(t_{k}-\tau_{j}\right)\right]^{2} \\
& \quad \leq L_{\psi}\left(s-t_{k}\right)^{2} \\
& \quad \leq L_{\psi} h^{2} .
\end{aligned}
$$

If $t_{k}-\tau_{j} \leq 0$ and $s-\tau_{j}>0$, with (13) we obtain

$$
\begin{aligned}
& E\left[x_{j}\left(s-\tau_{j}\right)-\widetilde{Z}_{j}\left(t_{k}\right)\right]^{2} \\
&= E\left[x_{j}\left(s-\tau_{j}\right)-\psi_{j}\left(t_{k}-\tau_{j}\right)\right]^{2} \\
& \leq 2 E\left[x_{j}\left(s-\tau_{j}\right)-x_{j}(0)\right]^{2} \\
&+2 E\left[\psi_{j}(0)-\psi_{j}\left(t_{k}-\tau_{j}\right)\right]^{2} \\
& \leq 2\left(\Gamma_{2}+L_{\psi} h\right) h .
\end{aligned}
$$

If $t_{k}-\tau_{j}>0$ and $s-\tau_{j}>0$, we assume $t_{k}-\tau_{j} \in\left[t_{k-q_{j}}, t_{k-q_{j}+1}\right)$ without loss of generality. Hence,

$$
\begin{aligned}
E\left[x_{j}\left(s-\tau_{j}\right)-\widetilde{Z}_{j}\left(t_{k}\right)\right]^{2} \\
=E\left[x_{j}\left(s-\tau_{j}\right)-x_{j}\left(t_{k-q_{j}}\right)\right]^{2} \\
\leq 2 E\left[x_{j}\left(s-\tau_{j}\right)-x_{j}\left(t_{k}-\tau_{j}\right)\right]^{2} \\
\quad+2 E\left[x_{j}\left(t_{k-q_{j}}\right)-x_{j}\left(t_{k}-\tau_{j}\right)\right]^{2} \\
\leq 4 \Gamma_{2} h
\end{aligned}
$$

by using inequality (13).

Lemma 5. Let $\mathbf{x}(t)$ denote the exact solution of (1). One assumes conditions (6) and (7). Then for the local intermediate value $\mathbf{y}\left(t_{k}\right):=\mathbf{x}\left(t_{k}\right)-(I+\theta C h)^{-1} C h \mathbf{x}\left(t_{k}\right)+(I+$ $\theta C h)^{-1} h\left[A \mathbf{f}\left(\mathbf{x}\left(t_{k}\right)\right)+B \mathbf{g}\left(\widetilde{Z}\left(t_{k}\right)\right)\right]$, one has the estimation

$$
E\left(\left\|\mathbf{x}\left(t_{k}\right)-\mathbf{y}\left(t_{k}\right)\right\|^{2}\right) \leq \Gamma_{3} h^{2} .
$$

Proof. The difference between the $i$ th components of $\mathbf{x}\left(t_{k}\right)$ and $\mathbf{y}\left(t_{k}\right)$ leads to

$$
\left|x_{i}\left(t_{k}\right)-y_{i}\left(t_{k}\right)\right|^{2}
$$

$$
=\frac{h^{2}}{\left(1+c_{i} \theta h\right)^{2}}\left[c_{i} x_{i}\left(t_{k}\right)-\sum_{j=1}^{n} a_{i j} f_{j}\left(x_{j}\left(t_{k}\right)\right)\right.
$$

$$
\left.-\sum_{j=1}^{n} b_{i j} g_{j}\left(\widetilde{Z}_{j}\left(t_{k}\right)\right)\right]^{2}
$$


whose expectation, together with $c_{i}>0, f_{i}(0)=g_{i}(0)=0$, the Lipschitz condition (6), and the estimation (12), gives

$$
\begin{gathered}
E\left(\left\|\mathbf{x}\left(t_{k}\right)-\mathbf{y}\left(t_{k}\right)\right\|^{2}\right) \\
\leq \sum_{i=1}^{n} \frac{3 h^{2}}{\left(1+c_{i} \theta h\right)^{2}} \\
\quad \times\left[c_{i}^{2} E\left|x_{i}\left(t_{k}\right)\right|^{2}+n \sum_{j=1}^{n} a_{i j}^{2} \alpha_{j}^{2} E\left|x_{j}\left(t_{k}\right)\right|^{2}\right. \\
\left.\quad+n \sum_{j=1}^{n} b_{i j}^{2} \beta_{j}^{2} E\left|\widetilde{Z}_{j}\left(t_{k}\right)\right|^{2}\right] \\
\leq 3 \Gamma_{\psi} \sum_{i=1}^{n}\left(c_{i}^{2}+n \sum_{j=1}^{n} a_{i j}^{2} \alpha_{j}^{2}+n \sum_{j=1}^{n} b_{i j}^{2} \beta_{j}^{2}\right) h^{2} .
\end{gathered}
$$

Now we discuss local error estimates.

Theorem 6. When one assumes Assumptions 1 and 2 and the conditions of Lemma 3, there exist positive constants $\Gamma_{0}$ and $\Gamma_{1}$, such that

$$
\begin{aligned}
& \max _{0 \leq k \leq K-1}\left\|E\left(\delta^{k+1}\right)\right\| \leq \Gamma_{0} h^{2}, \\
& \max _{0 \leq k \leq K-1} E\left(\left\|\delta^{k+1}\right\|^{2}\right) \leq \Gamma_{1} h^{3}
\end{aligned}
$$

as $h \downarrow 0$.

Proof. The Itô integral form of the $i$ th component of (1) on $\left[t_{k}, t\right]$ implies

$$
\begin{aligned}
x_{i}(t)- & x_{i}\left(t_{k}\right) \\
= & \int_{t_{k}}^{t}\left[-c_{i} x_{i}(s)+\sum_{j=1}^{n} a_{i j} f_{j}\left(x_{j}(s)\right)\right. \\
& \left.\quad+\sum_{j=1}^{n} b_{i j} g_{j}\left(x_{j}\left(s-\tau_{j}\right)\right)\right] \mathrm{d} s \\
& +\int_{t_{k}}^{t} \phi_{i}\left(x_{i}(s)\right) \mathrm{d} W_{i}(s) .
\end{aligned}
$$

By utilizing the previous identity, the $i$ th component of the difference $\delta^{k+1}$ introduced in Definition 1 can be calculated as

$$
\begin{aligned}
\delta_{i}^{k+1}= & x_{i}\left(t_{k+1}\right)-x_{i}\left(t_{k}\right)+\frac{c_{i} h}{1+c_{i} \theta h} x_{i}\left(t_{k}\right) \\
& -\frac{h}{1+c_{i} \theta h}\left[\sum_{j=1}^{n} a_{i j} f_{j}\left(x_{j}\left(t_{k}\right)\right)+\sum_{j=1}^{n} b_{i j} g_{j}\left(\widetilde{Z}_{j}\left(t_{k}\right)\right)\right] \\
& -\phi_{i}\left(y_{i}\left(t_{k}\right)\right) \Delta W_{i}^{k}-\phi_{i}^{\prime}\left(y_{i}\left(t_{k}\right)\right) \\
& \times \phi_{i}\left(y_{i}\left(t_{k}\right)\right) \frac{\left(\Delta W_{i}^{k}\right)^{2}-h}{2}
\end{aligned}
$$

$$
\begin{aligned}
= & \int_{t_{k}}^{t_{k+1}} c_{i}\left[x_{i}\left(t_{k}\right)-x_{i}(s)\right] \mathrm{d} s-\frac{c_{i}^{2} h^{2} \theta}{1+c_{i} \theta h} x_{i}\left(t_{k}\right) \\
& +\int_{t_{k}}^{t_{k+1}} \sum_{j=1}^{n} a_{i j}\left[f_{j}\left(x_{j}(s)\right)-f_{j}\left(x_{j}\left(t_{k}\right)\right)\right] \\
& +\sum_{j=1}^{n} b_{i j}\left[g_{j}\left(x_{j}\left(s-\tau_{j}\right)\right)-g_{j}\left(\widetilde{Z}_{j}\left(t_{k}\right)\right)\right] \mathrm{d} s \\
& +\frac{c_{i} \theta h^{2}}{1+c_{i} \theta h}\left[\sum_{j=1}^{n} a_{i j} f_{j}\left(x_{j}\left(t_{k}\right)\right)+\sum_{j=1}^{n} b_{i j} g_{j}\left(\widetilde{Z}_{j}\left(t_{k}\right)\right)\right] \\
& +\int_{t_{k}}^{t_{k+1}} \phi_{i}\left(x_{i}(s)\right) \mathrm{d} W_{i}(s)-\int_{t_{k}}^{t_{k+1}} \phi_{i}\left(y_{i}\left(t_{k}\right)\right) \mathrm{d} W_{i}(s) \\
& -\int_{t_{k}}^{t_{k+1}} \int_{t_{k}}^{s} \phi_{i}^{\prime}\left(y_{i}\left(t_{k}\right)\right) \phi_{i}\left(y_{i}\left(t_{k}\right)\right) \mathrm{d} W_{i}(r) \mathrm{d} W_{i}(s),
\end{aligned}
$$

where $\Delta W_{i}^{k}=\int_{t_{k}}^{t_{k+1}} \mathrm{~d} W_{i}(s)$ and $\left(\left(\Delta W_{\mathrm{i}}^{k}\right)^{2}-h\right) / 2=$ $\int_{t_{k}}^{t_{k+1}} \int_{t_{k}}^{s} \mathrm{~d} W_{i}(r) \mathrm{d} W_{i}(s)$.

Taking expectations of both sides of (25),

$$
\begin{aligned}
& E\left(\delta_{i}^{k+1}\right) \\
& =E\left(\int_{t_{k}}^{t_{k+1}} c_{i} \int_{t_{k}}^{s}-\mu_{i}\left(x_{i}(r)\right) \mathrm{d} r \mathrm{~d} s\right) \\
& -\frac{c_{i}^{2} h^{2} \theta}{1+c_{i} \theta h} E\left(x_{i}\left(t_{k}\right)\right) \\
& +E\left(\int_{t_{k}}^{t_{k+1}} \sum_{j=1}^{n} a_{i j} \int_{t_{k}}^{s} f_{j}^{\prime}\left(x_{j}(r)\right) \mu_{j}\left(x_{j}(r)\right)\right. \\
& \left.+\frac{1}{2} f_{j}^{\prime \prime}\left(x_{j}(r)\right) \phi_{j}^{2}\left(x_{j}(r)\right) \mathrm{d} r \mathrm{~d} s\right) \\
& +E\left(\int_{t_{k}}^{t_{k+1}} \sum_{j=1}^{n} b_{i j} \int_{t_{k-q_{j}}}^{s-\tau_{j}} g_{j}^{\prime}\left(x_{j}(r)\right) \mu_{j}\left(x_{j}(r)\right)\right. \\
& \left.+\frac{1}{2} g_{j}^{\prime \prime}\left(x_{j}(r)\right) \phi_{j}^{2}\left(x_{j}(r)\right) \mathrm{d} r \mathrm{~d} s\right) \\
& +\frac{c_{i} \theta h^{2}}{1+c_{i} \theta h}\left[\sum_{j=1}^{n} a_{i j} E\left(f_{j}\left(x_{j}\left(t_{k}\right)\right)\right)\right. \\
& \left.+\sum_{j=1}^{n} b_{i j} E\left(g_{j}\left(\widetilde{Z}_{j}\left(t_{k}\right)\right)\right)\right]
\end{aligned}
$$

by (24) and Itô formula, where $\mu_{i}\left(x_{i}(r)\right):=-c_{i} x_{i}(r)+$ $\sum_{j=1}^{n} a_{i j} f_{j}\left(x_{j}(r)\right)+\sum_{j=1}^{n} b_{i j} g_{j}\left(x_{j}\left(r-\tau_{j}\right)\right)$. Under conditions of this theorem, we have $\left|E\left(\delta_{i}^{k+1}\right)\right| \leq \widetilde{\Gamma}_{0} h^{2}$ by (14), Jensen 
inequality $|E(X)| \leq E(|X|)$, triangle inequality, and properties of definite integral. Then we have $\max _{0 \leq k \leq K-1}\left\|E\left(\delta^{k+1}\right)\right\| \leq$ $\Gamma_{0} h^{2}$ from the relation between $\left|E\left(\delta_{i}^{k+1}\right)\right|$ and $\left\|E\left(\delta^{k+1}\right)\right\|$.

Now we prove (23). By Itô formula,

$$
\begin{gathered}
\int_{t_{k}}^{t_{k+1}} \phi_{i}\left(x_{i}(s)\right) \mathrm{d} W_{i}(s)-\int_{t_{k}}^{t_{k+1}} \phi_{i}\left(y_{i}\left(t_{k}\right)\right) \mathrm{d} W_{i}(s) \\
\quad-\int_{t_{k}}^{t_{k+1}} \int_{t_{k}}^{s} \phi_{i}^{\prime}\left(y_{i}\left(t_{k}\right)\right) \phi_{i}\left(y_{i}\left(t_{k}\right)\right) \mathrm{d} W_{i}(r) \mathrm{d} W_{i}(s) \\
=\int_{t_{k}}^{t_{k+1}}\left[\phi_{i}\left(x_{i}\left(t_{k}\right)\right)-\phi_{i}\left(y_{i}\left(t_{k}\right)\right)\right] \mathrm{d} W_{i}(s) \\
+\int_{t_{k}}^{t_{k+1}} \int_{t_{k}}^{s} \phi_{i}^{\prime}\left(x_{i}(r)\right) \mu_{i}\left(x_{i}(r)\right) \\
+\frac{1}{2} \phi_{i}^{\prime \prime}\left(x_{i}(r)\right) \phi_{i}\left(x_{i}(r)\right)^{2} \mathrm{~d} r \mathrm{~d} W_{i}(s) \\
+\int_{t_{k}}^{t_{k+1}} \int_{t_{k}}^{s}\left[\phi_{i}^{\prime}\left(x_{i}(r)\right) \phi_{i}\left(x_{i}(r)\right)-\phi_{i}^{\prime}\left(y_{i}\left(t_{k}\right)\right)\right. \\
\left.\times \phi_{i}\left(y_{i}\left(t_{k}\right)\right)\right] \mathrm{d} W_{i}(r) \mathrm{d} W_{i}(s) .
\end{gathered}
$$

From (25) and (27), we have

$$
\begin{aligned}
& E\left(\left|\delta_{i}^{k+1}\right|^{2}\right) \\
& \leq 9\{\underbrace{c_{i} h \int_{t_{k}}^{t_{k+1}} E\left[x_{i}\left(t_{k}\right)-x_{i}(s)\right]^{2} \mathrm{~d} s}_{\text {Cauchy-Schwartz inequality }} \\
& +\underbrace{n \sum_{j=1}^{n} a_{i j}^{2} \alpha_{j}^{2} h \int_{t_{k}}^{t_{k+1}} E\left[x_{j}(s)-x_{j}\left(t_{k}\right)\right]^{2} \mathrm{~d} s}_{\text {Cauchy-Schwartz and Hölder inequality }} \\
& +\underbrace{n \sum_{j=1}^{n} b_{i j}^{2} \beta_{j}^{2} h \int_{t_{k}}^{t_{k+1}} E\left[x_{j}\left(s-\tau_{j}\right)-\widetilde{Z}_{j}\left(t_{k}\right)\right]^{2} \mathrm{~d} s}_{\text {Cauchy-Schwartz and Hölder inequality }} \\
& +\frac{c_{i}^{4} h^{4} \theta^{2}}{\left(1+c_{i} \theta h\right)^{2}} E\left|x_{i}\left(t_{k}\right)\right|^{2} \\
& +\frac{n c_{i}^{2} h^{4} \theta^{2}}{\left(1+c_{i} \theta h\right)^{2}}\left[\sum_{j=1}^{n} a_{i j}^{2} E\left|f_{j}\left(x_{j}\left(t_{k}\right)\right)\right|^{2}+\sum_{j=1}^{n} b_{i j}^{2} E\left|g_{j}\left(\widetilde{Z}_{j}\left(t_{k}\right)\right)\right|^{2}\right] \\
& +\underbrace{\int_{t_{k}}^{t_{k+1}} \gamma_{j}^{2} E\left[x_{i}\left(t_{k}\right)-y_{i}\left(t_{k}\right)\right]^{2} \mathrm{~d} s}_{\text {Itô Isometry }} \\
& \underbrace{\int_{t_{k}}^{t_{k+1}}\left(s-t_{k}\right) \int_{t_{k}}^{s} E\left|\phi_{i}^{\prime}\left(x_{i}(r)\right) \mu_{i}\left(x_{i}(r)\right)+(1 / 2) \phi_{i}^{\prime \prime}\left(x_{i}(r)\right) \phi_{i}\left(x_{i}(r)\right)^{2}\right|^{2} \mathrm{~d} r \mathrm{~d} s}_{\text {Cauchy-Schwartz inequality and Itô Isometry }} \\
& +\underbrace{\int_{t_{k}}^{t_{k+1}} \int_{t_{k}}^{s} E\left[\phi_{i}^{\prime}\left(x_{i}(r)\right) \phi_{\mathrm{i}}\left(x_{i}(r)\right)-\phi_{i}^{\prime}\left(y_{i}\left(t_{k}\right)\right) \phi_{i}\left(y_{i}\left(t_{k}\right)\right)\right]^{2} \mathrm{~d} r \mathrm{~d} s}_{\text {Itô Isometry }}\}
\end{aligned}
$$

$$
\begin{aligned}
\leq 9\{h\left(c_{i}+n \sum_{j=1}^{n} a_{i j}^{2} \alpha_{j}^{2}\right) \int_{t_{k}}^{t_{k+1}} \underbrace{\Gamma_{2} h \mathrm{~d} s}_{(13)} \\
+h\left(n \sum_{j=1}^{n} b_{i j}^{2} \beta_{j}^{2}\right) \int_{t_{k}}^{t_{k+1}} \underbrace{\Gamma_{\tau} h \mathrm{~d} s}_{(15)} \\
+\frac{h^{4} c_{i}^{2} \theta^{2}}{\left(1+c_{i} \theta h\right)^{2}} \underbrace{\Gamma_{\psi}\left[c_{i}^{2}+n \sum_{j=1}^{n} a_{i j}^{2} \alpha_{j}^{2}+n \sum_{j=1}^{n} b_{i j}^{2} \beta_{j}^{2}\right]}_{\left(\Gamma_{\psi}\right.} \\
+\int_{t_{k}}^{t_{k+1}} \gamma_{j}^{2} \underbrace{\Gamma_{3} h^{2}}_{(19)} \mathrm{d} s+\int_{t_{k}}^{t_{k+1}} \underbrace{\Gamma_{4}\left(s-t_{k}\right)^{2} \mathrm{~d} s}_{\text {moment bounded }} \\
+\underbrace{\int_{t_{k}}^{t_{k+1}} \int_{t_{k}}^{s} \Gamma_{5} h \mathrm{~d} r \mathrm{~d} s}_{\text {Triangle Inequality, (6), (13), (19) }}\} \leq \widetilde{\Gamma}_{1} h^{3} .
\end{aligned}
$$

Finally, it is easy to prove $E\left(\left\|\delta^{k+1}\right\|^{2}\right) \leq \Gamma_{1} h^{3}$.

Thanks to Theorem 1 in [18], we can conclude that

$$
E\left(\left\|\epsilon^{k}\right\|^{2}\right) \leq \Gamma h^{2} \quad \forall h \in(0,1),
$$

that is, the mean-square order of global error of the SSTM is 1.

\section{Stability of SSTM}

We are concerned with the stability of SSTM solution. Since (1) has an equilibrium solution $\mathbf{x}(t) \equiv \mathbf{0}$, we will discuss whether the SSTM solution $X_{k}$ with a positive step-size can attain a similar stability when $k$ goes to infinity. First we give a sufficient condition for the exponential stability in meansquare sense of the equilibrium solution. The references $[13$, 19 ] give the condition as

$$
\begin{gathered}
\sum_{j=1}^{n}\left|a_{i j}\right| \alpha_{j}+\sum_{j=1}^{n}\left|b_{i j}\right| \beta_{j} \leq \alpha_{i} \sum_{j=1}^{n}\left|a_{j i}\right|+\beta_{i} \sum_{j=1}^{n}\left|b_{j i}\right|, \\
-2 c_{i}+\sum_{j=1}^{n}\left|a_{i j}\right| \alpha_{j}+\sum_{j=1}^{n}\left|b_{i j}\right| \beta_{j}+\alpha_{i} \sum_{j=1}^{n}\left|a_{j i}\right|+\beta_{i} \sum_{j=1}^{n}\left|b_{j i}\right|+\gamma_{i}^{2}<0
\end{gathered}
$$

for every $i(i=1, \ldots, n)$.

Definition 7. A numerical method is said to be mean-square stable (MS-stable) if there exists an $h_{0}>0$ such that any application of the method to problem (1) generates numerical approximations $X_{i}^{k}$, which satisfies

$$
\lim _{k \rightarrow \infty} E\left(\left|X_{i}^{k}\right|^{2}\right)=0, \quad i=1,2, \ldots, n,
$$

for all $h \in\left(0, h_{0}\right)$.

Theorem 8. Assume (6), (30), and $(1-\theta) c_{i} h \leq 1$ are satisfied; then the SSTM (2) are mean-square stable if $h \in\left(0, h_{0}\right)$, where

$$
h_{0}= \begin{cases}\min _{1 \leq i \leq n}\left\{1, h_{i}\right\} & \text { for } \theta=1, \\ \min _{1 \leq i \leq n}\left\{1, \frac{1}{(1-\theta) c_{i}}, h_{i}\right\} & \text { for } \theta \in[0,1) .\end{cases}
$$


Here $h_{i}$ is the smallest positive root of the cubic equation with respect to $z$ given by

$$
\mathscr{A}_{i} z^{3}+\mathscr{B}_{i} z^{2}+\mathscr{C}_{i} z+\mathscr{D}_{i}=0,
$$

where the coefficients mean

$$
\begin{aligned}
\mathscr{A}_{i}= & \frac{\kappa_{i}^{2}}{2}\left(-(1-\theta) c_{i}+\sum_{j=1}^{n}\left|a_{i j}\right| \alpha_{j}+\sum_{j=1}^{n}\left|b_{i j}\right| \beta_{j}\right)^{2}, \\
\mathscr{B}_{i}= & \gamma_{i}^{2}\left(-(1-\theta) c_{i}+\sum_{j=1}^{n}\left|a_{i j}\right| \alpha_{j}+\sum_{j=1}^{n}\left|b_{i j}\right| \beta_{j}\right)^{2} \\
& +\kappa_{i}^{2}\left(\sum_{j=1}^{n}\left|a_{i j}\right| \alpha_{j}+\sum_{j=1}^{n}\left|b_{i j}\right| \beta_{j}\right)-\kappa_{i}^{2}(1-\theta) c_{i}, \\
\mathscr{C}_{i}= & \left.-(1-\theta) c_{i}+\sum_{j=1}^{n}\left|a_{i j}\right| \alpha_{j}+\sum_{j=1}^{n}\left|b_{i j}\right| \beta_{j}\right)^{2} \\
& +2 \gamma_{i}^{2}\left(-(1-\theta) c_{i}+\sum_{j=1}^{n}\left|a_{i j}\right| \alpha_{j}+\sum_{j=1}^{n}\left|b_{i j}\right| \beta_{j}\right) \\
& -c_{i}^{2} \theta^{2}+\frac{\kappa_{i}^{2}}{2}, \\
\mathscr{D}_{i}= & \left.-c_{i}+\sum_{j=1}^{n}\left|a_{i j}\right| \alpha_{j}+\sum_{j=1}^{n}\left|b_{i j}\right| \beta_{j}\right)+\gamma_{i}^{2} .
\end{aligned}
$$

for $i=1,2, \ldots, n$.

Squaring on both sides of (4b) and (4a), we have

$$
\begin{aligned}
\left|X_{i}^{k+1}\right|^{2}= & \left(Y_{i}^{k}\right)^{2}+\left[\phi_{i}\left(Y_{i}^{k}\right) \Delta W_{i}^{k}\right]^{2} \\
& +\left[\phi_{i}^{\prime}\left(Y_{i}^{k}\right) \phi_{i}\left(Y_{i}^{k}\right)\right]^{2}\left[\frac{\left(\Delta W_{i}^{k}\right)^{2}-h}{2}\right]^{2} \\
+ & {\left[\left(\phi_{i}\left(Y_{i}^{k}\right)\right) \Delta W_{i}^{k}+Y_{i}^{k}\right] } \\
\times & \phi_{i}^{\prime}\left(Y_{i}^{k}\right) \phi_{i}\left(Y_{i}^{k}\right)\left[\left(\Delta W_{i}^{k}\right)^{2}-h\right] \\
+ & 2 Y_{i}^{k} \phi_{i}\left(Y_{i}^{k}\right) \Delta W_{i}^{k}, \\
\left(1+\theta c_{i} h\right)^{2}\left|Y_{i}^{k}\right|^{2} & =\left[\left(1-(1-\theta) c_{i} h\right) X_{i}^{k}\right]^{2} \\
& +h^{2}\left[\sum_{j=1}^{n} a_{i j} f_{j}\left(X_{j}^{k}\right)\right]^{2} \\
& +h^{2}\left[\sum_{j=1}^{n} b_{i j} g_{j}\left(Z_{j}^{k}\right)\right]^{2}
\end{aligned}
$$

$$
\begin{aligned}
&+\sum_{j=1}^{n}\{ 2 h\left(1-(1-\theta) c_{i} h\right) X_{i}^{k} \\
& \times\left.\times\left[a_{i j} f_{j}\left(X_{j}^{k}\right)+b_{i j} g_{j}\left(Z_{j}^{k}\right)\right]\right\} \\
&+2 h^{2}\left[\sum_{j=1}^{n} a_{i j} f_{j}\left(X_{j}^{k}\right)\right]\left[\sum_{j=1}^{n} b_{i j} g_{j}\left(Z_{j}^{k}\right)\right] .
\end{aligned}
$$

Taking expectations of both sides of (35), we can get

$$
\begin{aligned}
E\left(\left|X_{i}^{k+1}\right|^{2}\right) & \\
= & E\left(\left|Y_{i}^{k}\right|^{2}\right)+h E\left(\left|\phi_{i}\left(Y_{i}^{k}\right)\right|^{2}\right) \\
& +\frac{h^{2}}{2} E\left(\left|\phi_{i}^{\prime}\left(Y_{i}^{k}\right) \phi_{i}\left(Y_{i}^{k}\right)\right|^{2}\right) \\
\leq & \left(1+\gamma_{i}^{2} h+\frac{h^{2}}{2} \kappa_{i}^{2}\right) E\left(\left|Y_{i}^{k}\right|^{2}\right) .
\end{aligned}
$$

Together with (36), we have

$$
\begin{aligned}
E\left(\left|X_{i}^{k+1}\right|^{2}\right) & \\
\leq & \left(1+\gamma_{i}^{2} h+\frac{h^{2}}{2} \kappa_{i}^{2}\right) E\left(\left|Y_{i}^{k}\right|^{2}\right) \\
\leq & \frac{1+\gamma_{i}^{2} h+\left(h^{2} / 2\right) \kappa_{i}^{2}}{\left(1+\theta c_{i} h\right)^{2}} \\
\times & \left\{\begin{array}{l}
{\left[1-(1-\theta) c_{i} h\right]^{2} E\left(\left|X_{i}^{k}\right|^{2}\right)} \\
+
\end{array}\right. \\
& +h^{2}\left(\sum_{r=1}^{n}\left|a_{i r}\right| \alpha_{r}\right)\left(\sum_{j=1}^{n}\left|a_{i j}\right| \alpha_{j} E\left(\left|X_{j}^{k}\right|^{2}\right)\right) \\
& +2 h^{2}\left(\sum_{i=1}^{n}\left|a_{i j}\right| \beta_{r}\right)\left(\sum_{j=1}^{n}\left|b_{i j}\right| \beta_{j} E\left(\left|Z_{j}^{k}\right|^{2}\right)\right) \\
& +\sum_{j=1}^{n} h\left|1-(1-\theta) c_{i} h\right| \\
& \times\left[\left|a_{i j}\right| \alpha_{j}\left[E\left(\left|X_{i j}^{k}\right|^{2}\right)+E\left(\left|X_{j}^{k}\right|^{2}\right)\right]\right.
\end{aligned}
$$


Thus, we attain

$$
\begin{aligned}
E\left(\left|X_{i}^{k+1}\right|^{2}\right) \leq & {\left[P(h)+\sum_{j=1}^{n} Q_{j}(h)+\sum_{j=1}^{n} R_{j}(h)\right] } \\
& \times \max _{1 \leq j \leq n}\left\{E\left(\left|X_{i}^{k}\right|^{2}\right), E\left(\left|X_{j}^{k}\right|^{2}\right), E\left(\left|Z_{j}^{k}\right|^{2}\right)\right\},
\end{aligned}
$$

where

$$
\begin{aligned}
& P(h)=\frac{1+\gamma_{i}^{2} h+\left(h^{2} \kappa_{i}^{2} / 2\right)}{\left(1+\theta c_{i} h\right)^{2}} \\
& \times\left\{\left[1-(1-\theta) c_{i} h\right]^{2}+h \sum_{j=1}^{n}\left|1-(1-\theta) c_{i} h\right|\right. \\
& \left.\times\left(\left|a_{i j}\right| \alpha_{j}+\left|b_{i j}\right| \beta_{j}\right)\right\}, \\
& Q_{j}(h)=\frac{1+\gamma_{i}^{2} h+\left(h^{2} \kappa_{i}^{2} / 2\right)}{\left(1+\theta c_{i} h\right)^{2}} \\
& \times\left\{h^{2}\left|a_{i j}\right| \alpha_{j} \sum_{j=1}^{n}\left(\left|a_{i r}\right| \alpha_{r}+\left|b_{i r}\right| \beta_{r}\right)\right. \\
& \left.+h\left|1-(1-\theta) c_{i} h\right|\left(\left|a_{i j}\right| \alpha_{j}\right)\right\} \\
& R_{j}(h)=\frac{1+\gamma_{i}^{2} h+\left(h^{2} \kappa_{i}^{2} / 2\right)}{\left(1+\theta c_{i} h\right)^{2}} \\
& \times\left\{h^{2}\left(\left|a_{i j}\right| \alpha_{j}+\left|b_{i j}\right| \beta_{j}\right)\left(\sum_{r=1}^{n}\left|b_{i r}\right| \beta_{r}\right)\right. \\
& \left.+h\left|1-(1-\theta) c_{i} h\right|\left(\left|b_{i j}\right| \beta_{j}\right)\right\} \text {. }
\end{aligned}
$$

Note that the assumption of Theorem implies the nonnegativity of $1-(1-\theta) c_{i} h$.

Proof. Obviously, when $k \rightarrow+\infty, E\left(\left|X_{i}^{k}\right|^{2}\right) \rightarrow 0$ if the inequality $P(h)+\sum_{j=1}^{n} Q_{j}(h)+\sum_{j=1}^{n} R_{j}(h)<1$ holds, which is equivalent to the inequality $\mathscr{A}_{i} h^{3}+\mathscr{B}_{i} h^{2}+\mathscr{C}_{i} h+\mathscr{D}_{i}<0$.

Furthermore, it is easy to prove $\mathscr{A}_{i}>0$ and $\mathscr{D}_{i}<0$ by virtue of (30). By Vieta's formulas, the product of three roots of (33) satisfies $z_{1} z_{2} z_{3}=-\left(\mathscr{D}_{i} / \mathscr{A}_{i}\right)>0$. This means that (33) has at least one positive root. Therefore, let $h_{i}$ denote the smallest positive root of the equation. Moreover, we note that at the origin the right-hand side polynomial of (33) is negative. This completes the proof.

\section{Numerical Results}

Now, we apply the introduced SSTM method to two test cases of SDHNNs in order to compare their performance with the split-step $\theta$-method in [13], which has strong convergence order 0.5 .

The mean-square error $\varepsilon$ of numerical approximations at time $T$ versus the step-size is depicted in log-log diagrams, where $\varepsilon:=\sqrt{(1 / 2000) \sum_{r=1}^{2000}\left\|X_{\omega_{r}}^{K}-\mathbf{x}(T)\right\|^{2}}$. Here $\mathbf{x}(T)$ stands for the value of explicit solution of (1) at time $T=3$ and $X_{\omega_{r}}^{K}$ is its numerical approximation along the $r$ th sample path $\left\{\omega_{r}\right.$ : $r=1,2, \ldots, 2000\}$. We compute the numerical solution using the split-step $\theta$-Milstein method (2) with step-size $h=2^{-12}$, and we will call this the "exact solution."

Example 9. Consider the following two-dimensional stochastic delay Hopfield neural networks of the form

$$
\begin{aligned}
d\left(\begin{array}{l}
x_{1}(t) \\
x_{2}(t)
\end{array}\right)= & -C\left(\begin{array}{l}
x_{1}(t) \\
x_{2}(t)
\end{array}\right) \mathrm{d} t+A\left(\begin{array}{l}
f\left(x_{1}(t)\right) \\
f\left(x_{2}(t)\right)
\end{array}\right) \mathrm{d} t \\
& +B\left(\begin{array}{l}
g\left(x_{1}\left(t-\tau_{1}\right)\right) \\
g\left(x_{2}\left(t-\tau_{2}\right)\right)
\end{array}\right) \mathrm{d} t+\Phi \mathrm{d} W(t)
\end{aligned}
$$

on $t \geqslant 0$ with the initial condition $x_{1}(t)=t+1, t \in[-1,0]$ and $x_{2}(t)=t+1, t \in[-2,0]$.

Case 1. Let $f(x)=\sin (x), g(x)=\arctan (x)$,

$$
\begin{gathered}
C=\left(\begin{array}{cc}
20 & 0 \\
0 & 20
\end{array}\right), \quad A=\left(\begin{array}{cc}
4 & -5 \\
6 & 3
\end{array}\right), \\
B=\left(\begin{array}{cc}
-6 & 4 \\
3 & 1
\end{array}\right), \quad \Phi=\left(\begin{array}{cc}
x_{1}(t) & 0 \\
0 & -\sqrt{5} x_{2}(t)
\end{array}\right) .
\end{gathered}
$$

Case 2. Let $f(x)=x, g(x)=\tanh (x)$,

$$
\begin{gathered}
C=\left(\begin{array}{cc}
10 & 0 \\
0 & 10
\end{array}\right), \quad A=\left(\begin{array}{ll}
0 & 0 \\
0 & 0
\end{array}\right), \\
B=\left(\begin{array}{cc}
5 & -1 \\
1 & 5
\end{array}\right), \quad \Phi=\left(\begin{array}{cc}
1.5 x_{1}(t) & 0 \\
0 & -1.5 x_{2}(t)
\end{array}\right) .
\end{gathered}
$$

In Figure 1, SSTM is applied with 7 different step-sizes: $h_{m}=2^{m-12}$ for $m=1,2, \ldots, 7$. Two pairs of time delays $\left(\tau_{1}, \tau_{2}\right)$ are set to $(1,2)$ and $(1.13,2.31)$. The first pair has common factor $h_{m}$; however, the second pair is incommensurable by $h_{m}$. The computation errors $\varepsilon$ versus step-sizes $h$ are plotted on a log-log scale and the reference lines of slope 1 are added. It illustrates that SSTM has raised the strong order of the split-step $\theta$-method at least to 1 for SDHNNs [13].

Next, Table 1 shows a comparison of stability intervals between the SST and the SSTM for (43). Two sets of the interval in the Table are calculated through Theorem 8 in this paper and Theorem 5.1 in [13]. It is easy to see that the stability intervals of the two methods are similar.

We know that Theorem 5.1 in [13] and Theorem 8 in this paper only give sufficient conditions of mean-square stability. Therefore the stability intervals given by these theorems are only subsets of real ones. To confirm the situation, we calculated the sample moments of the approximate solution and plotted them along the time $t$. Here the sample moment $\eta^{i}$ means $(1 / 2000) \sum_{r=1}^{2000}\left\|X_{\omega_{r}}^{i}\right\|^{2}$ for the numerical solution $X_{\omega_{r}}^{i}$ 


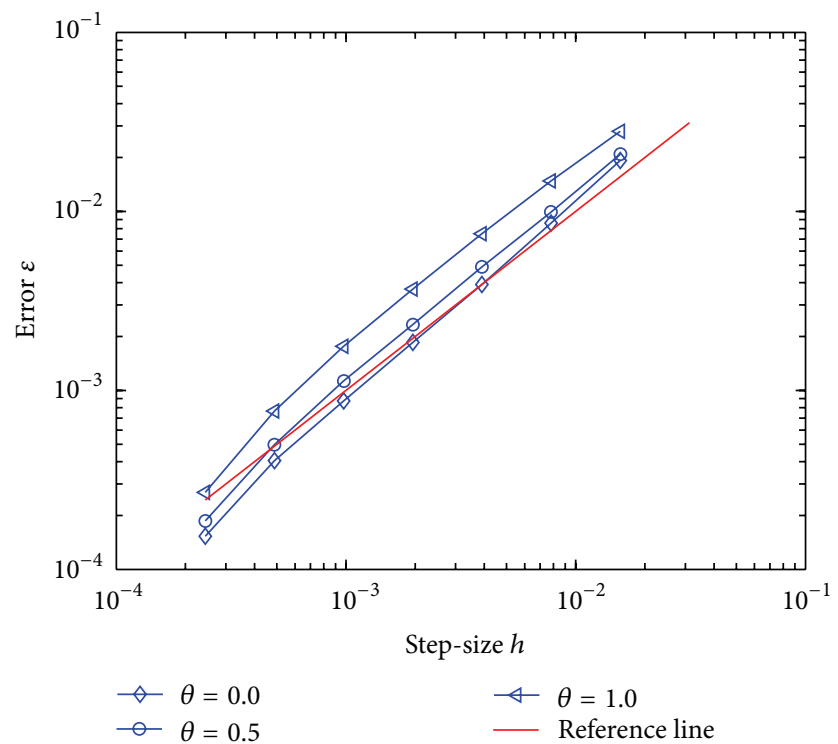

(a) Case 1 with $\tau_{1}=1, \tau_{2}=2$

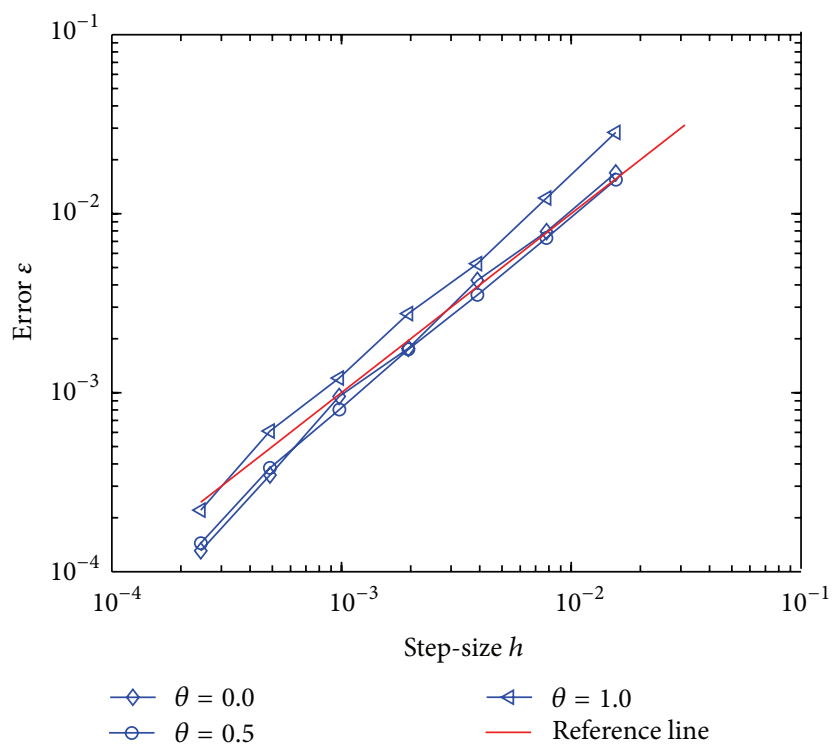

(c) Case 1 with $\tau_{1}=1.13, \tau_{2}=2.31$

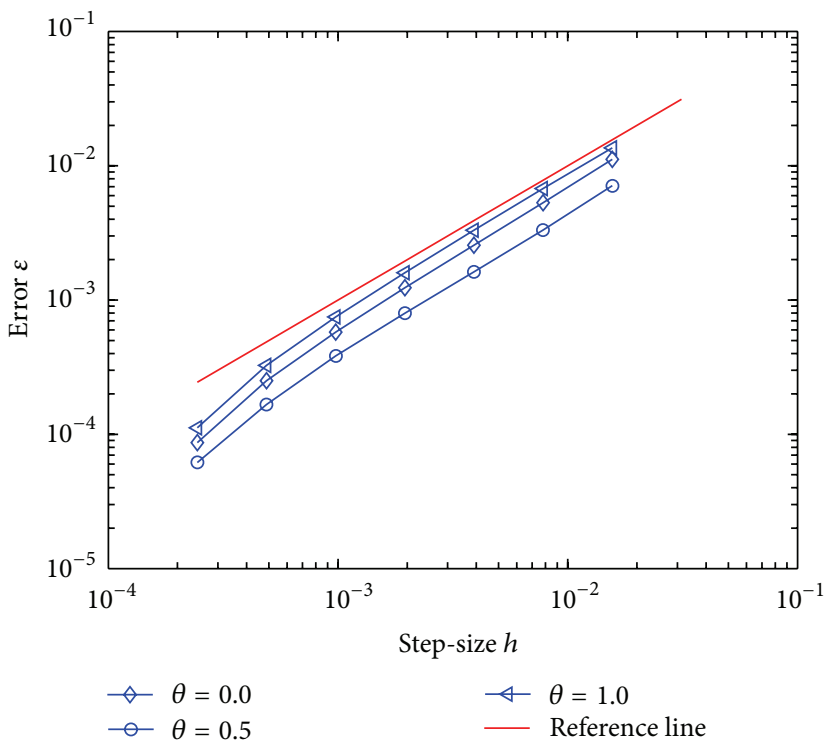

(b) Case 2 with $\tau_{1}=1, \tau_{2}=2$

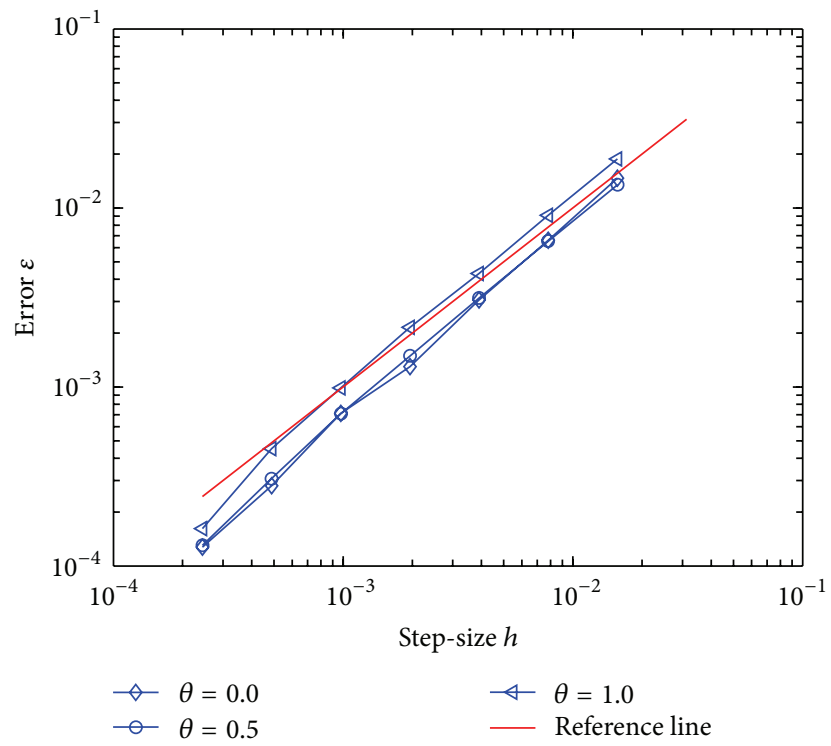

(d) Case 2 with $\tau_{1}=1.13, \tau_{2}=2.31$

FIGURE 1: Errors versus step-sizes for the SDHNNs (43).

TABLE 1: Calculated intervals of step-size for mean-square stability of the numerical schemes.

\begin{tabular}{lrr}
\hline Numerical scheme & Case 1 & Case 2 \\
\hline Split-step $\theta$-Milstein method $(\theta=0.0)$ & $(0.0000,0.0500]$ & $(0.0000,0.1000]$ \\
Split-step $\theta$-Milstein method $(\theta=0.2)$ & $(0.0000,0.0625]$ & $(0.0000,0.1250]$ \\
Split-step $\theta$-Milstein method $(\theta=0.5)$ & $(0.0000,0.1000]$ & $(0.0000,0.2000]$ \\
Split-step $\theta$-Milstein method $(\theta=0.8)$ & $(0.0000,0.0650)$ & $(0.0000,0.5000]$ \\
Split-step $\theta$-Milstein method $(\theta=1.0)$ & $(0.0000,0.0500)$ & $(0.0000,0.3534)$ \\
\hline Split-step $\theta$-method $(\theta=0.0)$ & $(0.0000,0.0500]$ & $(0.0000,0.1000]$ \\
Split-step $\theta$-method $(\theta=0.2)$ & $(0.0000,0.0625]$ & $(0.0000,0.1250]$ \\
Split-step $\theta$-method $(\theta=0.5)$ & $(0.0000,0.1000]$ & $(0.0000,0.2000]$ \\
Split-step $\theta$-method $(\theta=0.8)$ & $(0.0000,0.0689)$ & $(0.0000,0.5000]$ \\
Split-step $\theta$-method $(\theta=1.0)$ & $(0.0000,0.0540)$ & $(0.0000,0.5792)$ \\
\hline
\end{tabular}




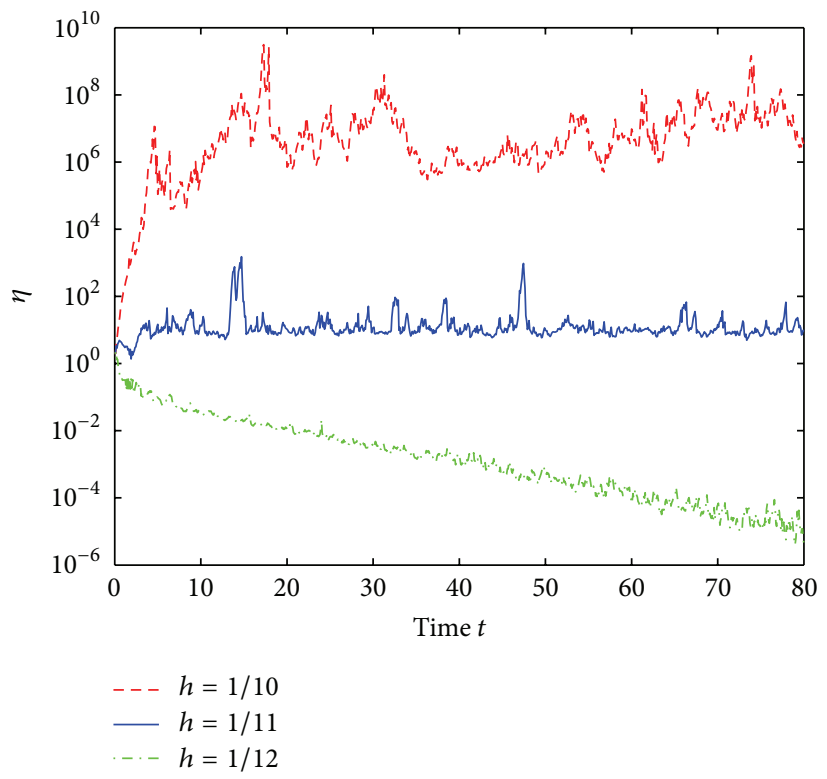

(a) SST

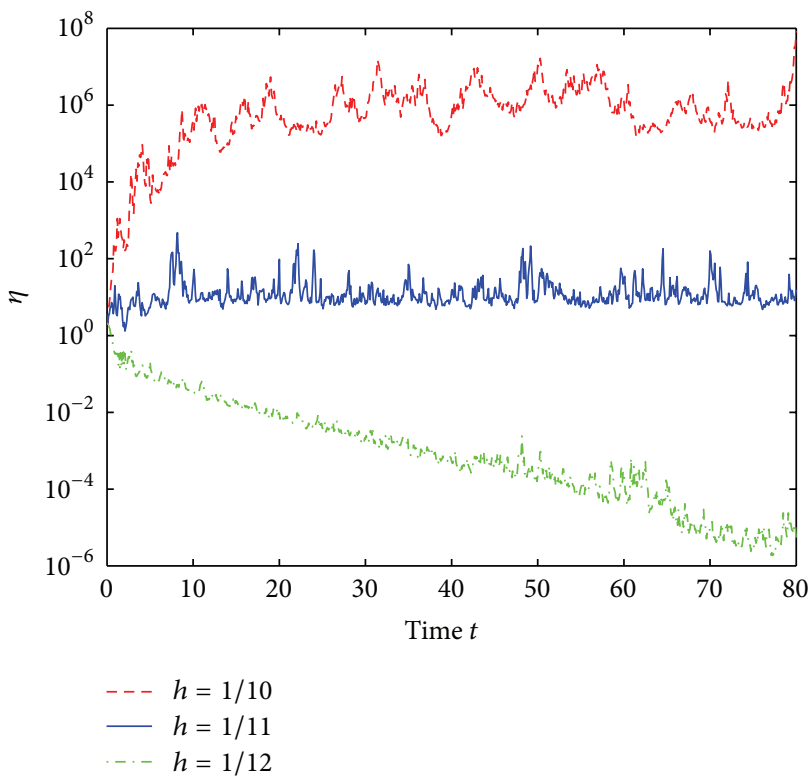

(b) SSTM

Figure 2: Mean-square stability comparison of SST and SSTM for the SDHNNs (43), Case 1, $\theta=0$.

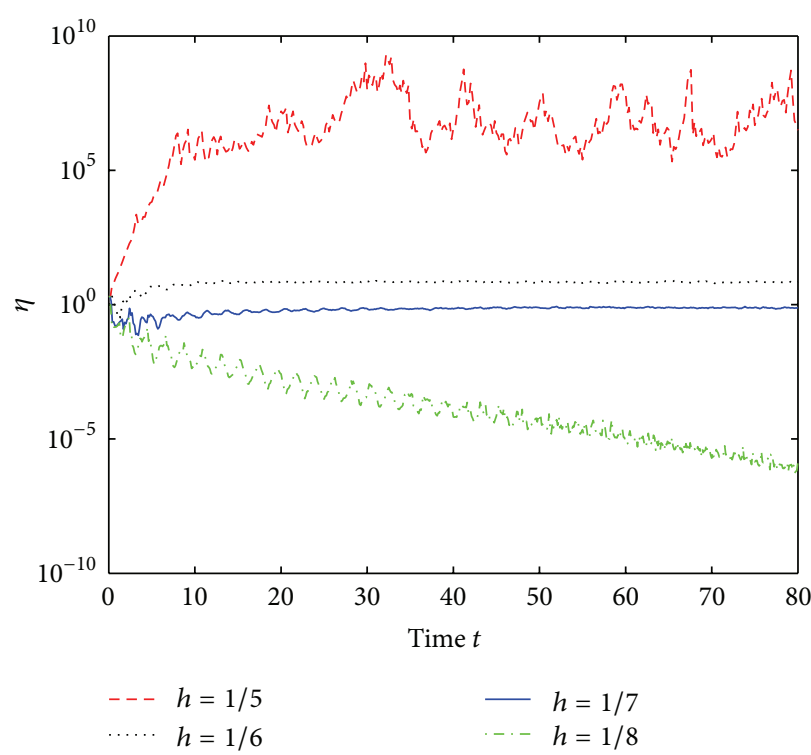

(a) SST

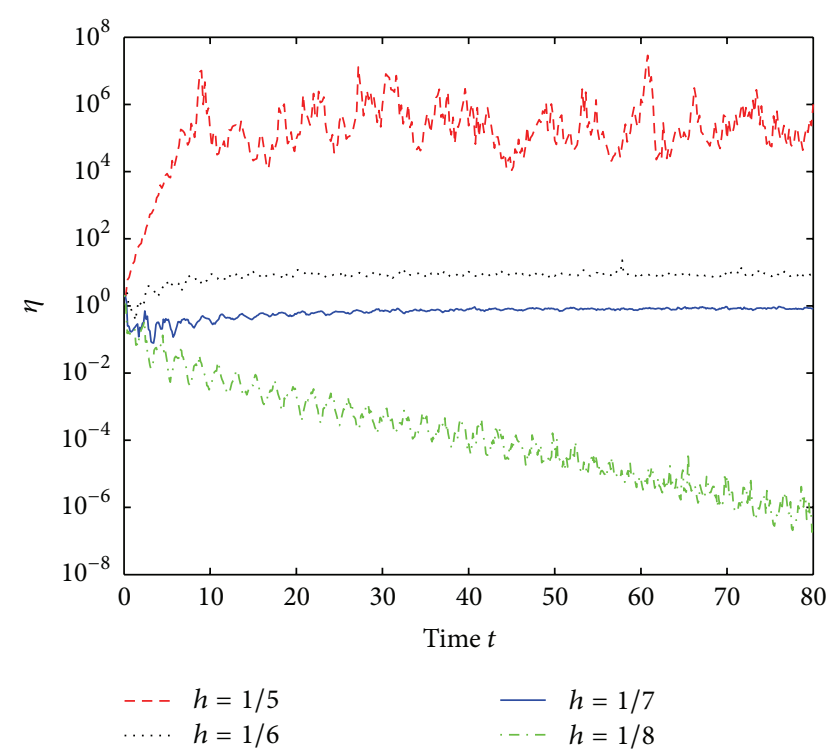

(b) SSTM

FIgURE 3: Mean-square stability comparison of SST and SSTM for the SDHNNs (43), Case 2, $\theta=0$.

approximating $\mathbf{x}\left(t_{i}\right)$ along the $r$ th sample path. Figures 2, 3, 4, 5 , and 6 depict the results by SST and SSTM in the log-scaled vertical axis. All the figures can give a rough estimate of the stability interval in each case.

\section{Concluding Remarks}

We introduce the split-step $\theta$-Milstein method (SSTM), which exhibits higher strong convergence rate than the split-step $\theta$-method (SST, see [13]) for a stochastic delay
Hopfield neural networks, and the scheme proposed in this paper can deal with incommensurable time delays which were not considered in [13]. We give the proof of convergence results, which has generally been omitted in the previous works on the same subject. By comparing the stability intervals of step size for the SST and SSTM for a test example, we find they exhibit similar mean-square stability.

In this paper, we have found a delay-independent sufficient condition for mean-square stability of split-step $\theta$-Milstein method applied to nonlinear stochastic delay 


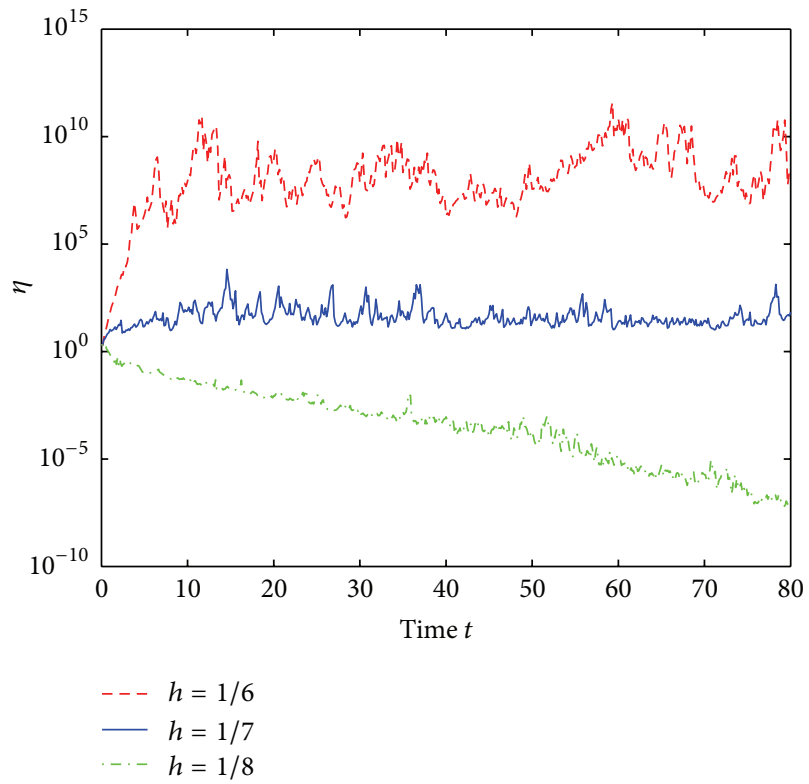

(a) SST

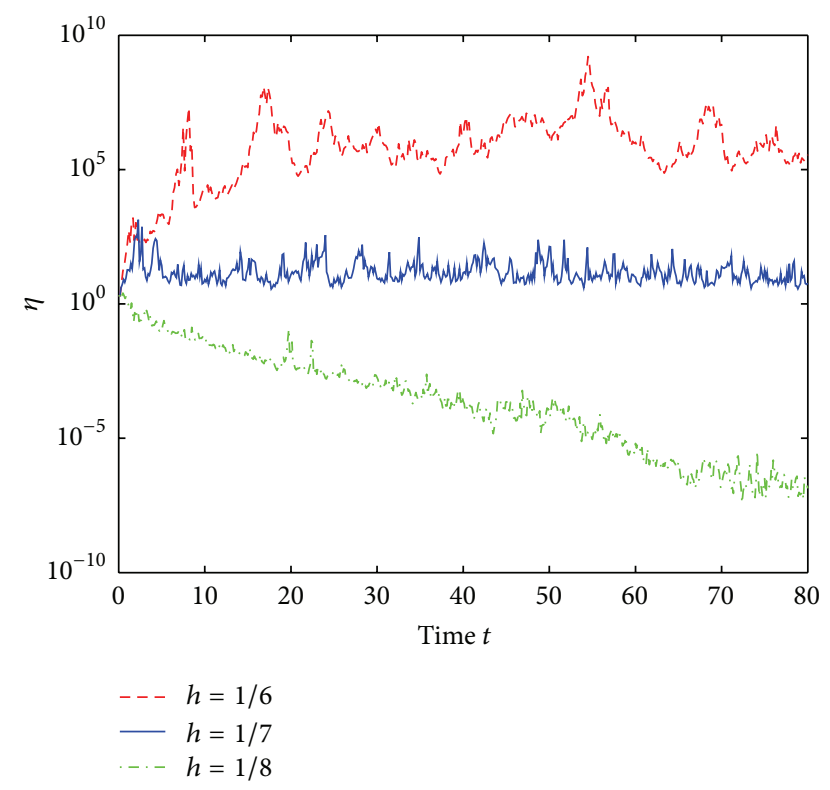

(b) SSTM

FIGURE 4: Mean-square stability comparison of SST and SSTM for the SDHNNs (43), Case 1, $\theta=0.2$.

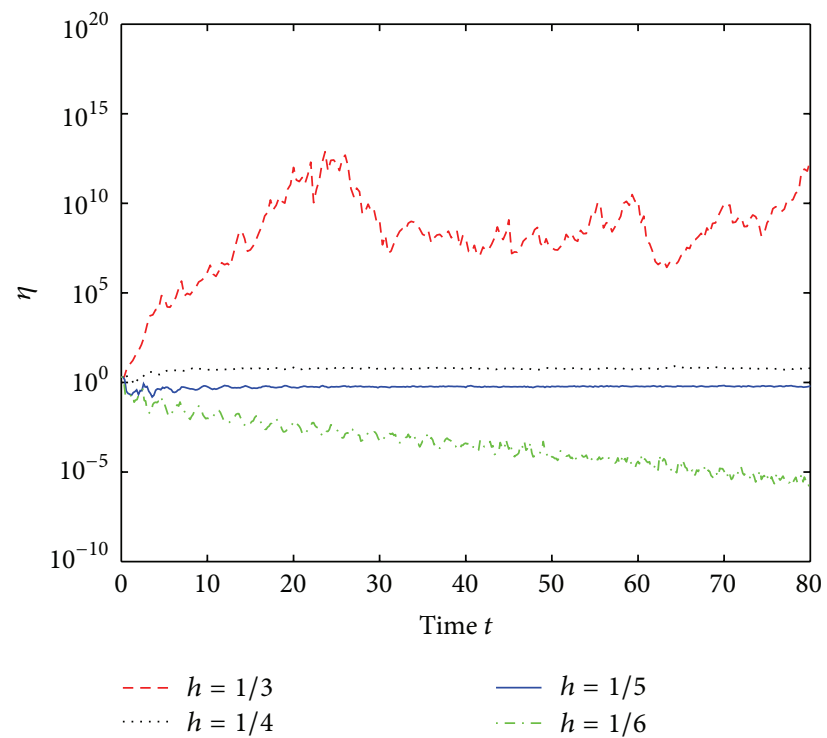

(a) SST

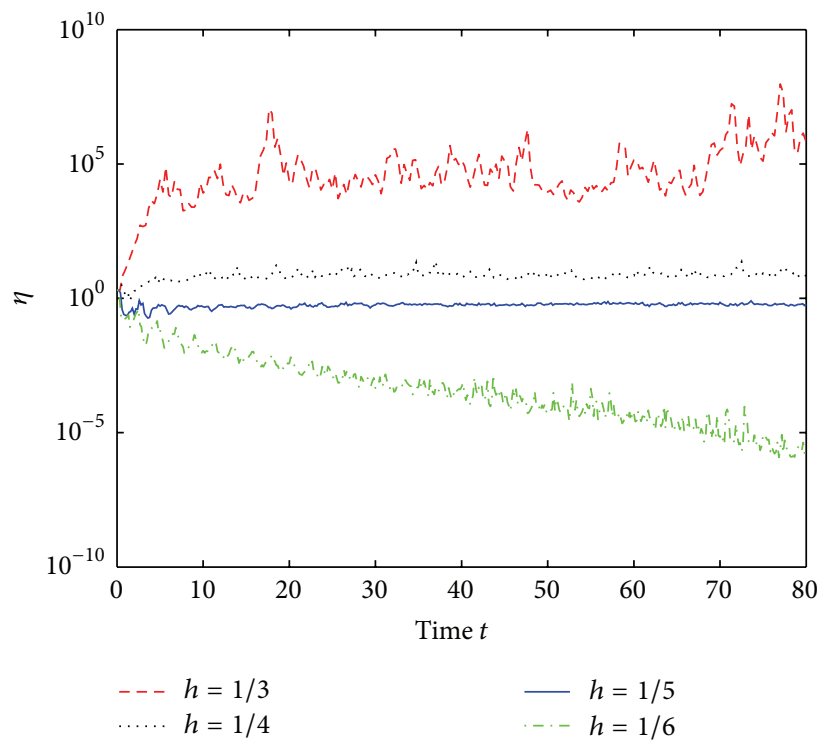

(b) SSTM

FIGURE 5: Mean-square stability comparison of SST and SSTM for the SDHNNs (43), Case 2, $\theta=0.2$.

Hopfield neural networks. Further, Figure 6 suggests that the value of $h_{0}$, the right end-point of the stability interval, given by Theorem 5.1 in [13] and Theorem 8 in this paper is much smaller than the true value when $\theta$ is close to unity. In this case, we need other techniques for stability analysis in this kind of stochastic delay differential system. To the best of our knowledge, the works in [20,21] put forward good attempts. On the other hand, with respect to stochastic delay differential equations, some other types of stability have been successfully discussed for the Euler-type scheme, for example, mean-square exponential stability [12], delay-dependent stability [22], delay-dependent exponential stability [23], and almost sure exponential stability [24]. To Milstein-type scheme, in view of more sophisticated derivations, these issues would be challenging for future research.

\section{Acknowledgment}

The authors would like to thank Dr. E. Buckwar and Dr. A. Rathinasamy for their valuable suggestions. The authors also thank the editor and the anonymous referees, whose 


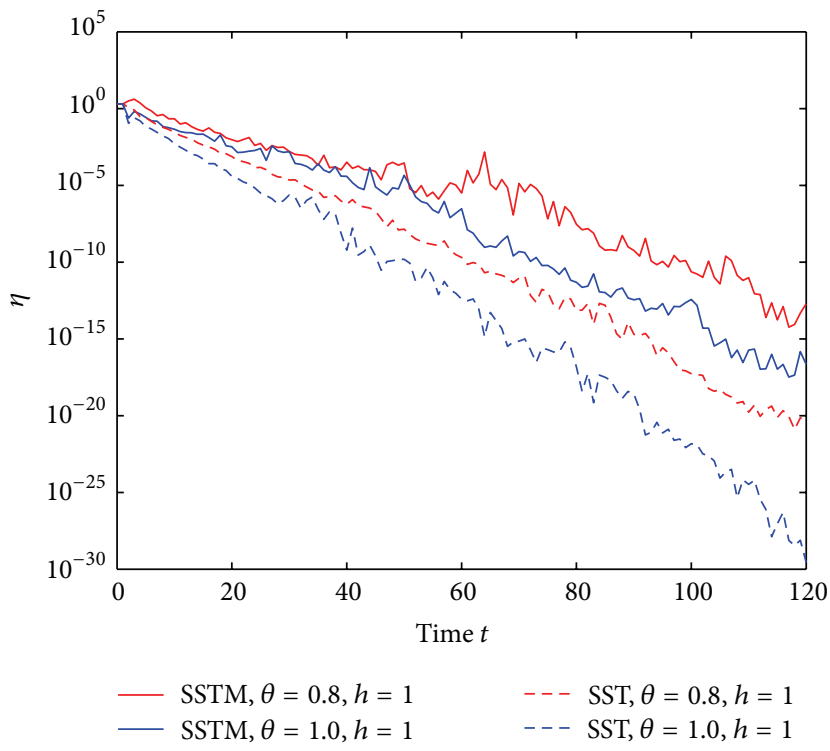

(a) Case 1

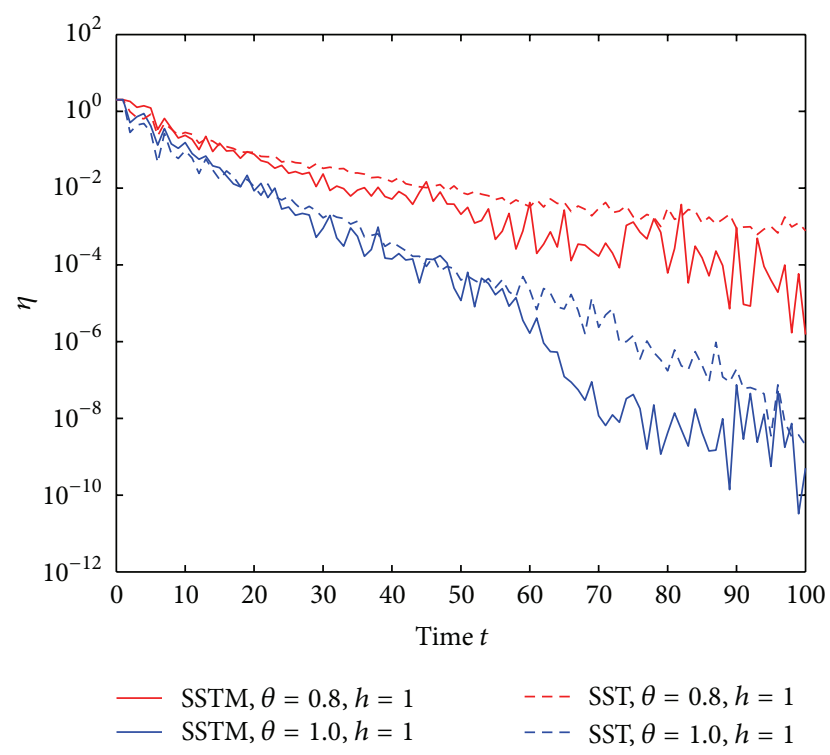

(b) Case 2

FIGURE 6: Mean-square stability comparison of SST and SSTM for the SDHNNs (43).

careful reading and constructive feedback have improved this work. The first author is partially supported by EInstitutes of Shanghai Municipal Education Commission (no. E03004), National Natural Science Foundation of China (no. 10901106), Natural Science Foundation of Shanghai (no. 09ZR1423200), and Innovation Program of Shanghai Municipal Education Commission (no. 09YZ150). The third author is partially supported by the Grants-in-Aid for Scientific Research (no. 24540154) supplied by Japan Society for the Promotion of Science.

\section{References}

[1] J. J. Hopfield, "Neural networks and physical systems with emergent collective computational abilities," Proceedings of the National Academy of Sciences of the United States of America, vol. 79, no. 8, pp. 2554-2558, 1982.

[2] J. J. Hopfield and D. W. Tank, "Neural computation of decisons in optimization problems," Biological Cybernetics, vol. 52, no. 3 , pp. 141-152, 1985.

[3] G. Joya, M. A. Atencia, and F. Sandoval, "Hopfield neural networks for optimization:study of the different dynamics," Neurocomputing, vol. 43, no. 1-4, pp. 219-237, 2002.

[4] Y. Sun, "Hopfield neural network based algorithms for image restoration and reconstruction. I. Algorithms and simulations," IEEE Transactions on Signal Processing, vol. 48, no. 7, pp. 21052118, 2000.

[5] S. Young, P. Scott, and N. Nasrabadi, "Object recognition using multilayer Hopfield neural network," IEEE Transactions on Image Processing, vol. 6, no. 3, pp. 357-372, 1997.

[6] G. Pajares, "A Hopfield neural network for image change detection," IEEE Transactions on Neural Networks, vol. 17, no. 5, pp. 1250-1264, 2006.
[7] L. Wan and J. H. Sun, "Mean square exponential stability of stochastic delayed Hopfield neural networks," Physics Letters A, vol. 343, no. 4, pp. 306-318, 2005.

[8] Z. Wang, H. Shu, J. Fang, and X. Liu, "Robust stability for stochastic Hopfield neural networks with time delays," Nonlinear Analysis, vol. 7, no. 5, pp. 1119-1128, 2006.

[9] Z. D. Wang, Y. R. Liu, K. Fraser, and X. H. Liu, "Stochastic stability of uncertain Hopfield neural networks with discrete and distributed delays," Physics Letters A, vol. 354, no. 4, pp. 288-297, 2006.

[10] J. S. Šíma and P. Orponen, "General-purpose computation with neural networks: a survey of complexity theoretic results," Neural Computation, vol. 15, no. 12, pp. 2727-2778, 2003.

[11] J. R. Raol and H. Madhuranath, "Neural network architectures for parameter estimation of dynamical systems," IEE Proceedings, vol. 143, no. 4, pp. 387-394, 1996.

[12] R. H. Li, W. Pang, and P. Leung, "Exponential stability of numerical solutions to stochastic delay Hopfield neural networks," Neurocomputing, vol. 73, no. 4-6, pp. 920-926, 2010.

[13] A. Rathinasamy, "The split-step $\theta$-methods for stochastic delay Hopfield neural networks," Applied Mathematical Modelling, vol. 36, no. 8, pp. 3477-3485, 2012.

[14] D. J. Higham, X. Mao, and A. M. Stuart, "Strong convergence of Euler-type methods for nonlinear stochastic differential equations," SIAM Journal on Numerical Analysis, vol. 40, no. 3, pp. 1041-1063, 2002.

[15] H. Zhang, S. Gan, and L. Hu, "The split-step backward Euler method for linear stochastic delay differential equations," Journal of Computational and Applied Mathematics, vol. 225, no. 2, pp. 558-568, 2009.

[16] X. Wang and S. Gan, "The improved split-step backward Euler method for stochastic differential delay equations," International Journal of Computer Mathematics, vol. 88, no. 11, pp. 2359-2378, 2011. 
[17] X. R. Mao, Stochastic Differential Equations and Applications, Horwood, Chichester, UK, 2nd edition, 2007.

[18] E. Buckwar, "One-step approximations for stochastic functional differential equations," Applied Numerical Mathematics, vol. 56, no. 5, pp. 667-681, 2006.

[19] Q. Zhou and L. Wan, "Exponential stability of stochastic delayed Hopfield neural networks," Applied Mathematics and Computation, vol. 199, no. 1, pp. 84-89, 2008.

[20] Y. Saito and T. Mitsui, "Mean-square stability of numerical schemes for stochastic differential systems," Vietnam Journal of Mathematics, vol. 30, pp. 551-560, 2002.

[21] E. Buckwar and C. Kelly, "Towards a systematic linear stability analysis of numerical methods for systems of stochastic differential equations," SIAM Journal on Numerical Analysis, vol. 48, no. 1, pp. 298-321, 2010.

[22] C. Huang, S. Gan, and D. Wang, "Delay-dependent stability analysis of numerical methods for stochastic delay differential equations," Journal of Computational and Applied Mathematics, vol. 236, no. 14, pp. 3514-3527, 2012.

[23] X. Qu and C. Huang, "Delay-dependent exponential stability of the backward Euler method for nonlinear stochastic delay differential equations," International Journal of Computer Mathematics, vol. 89, no. 8, pp. 1039-1050, 2012.

[24] F. Wu, X. Mao, and L. Szpruch, "Almost sure exponential stability of numerical solutions for stochastic delay differential equations," Numerische Mathematik, vol. 115, no. 4, pp. 681-697, 2010. 


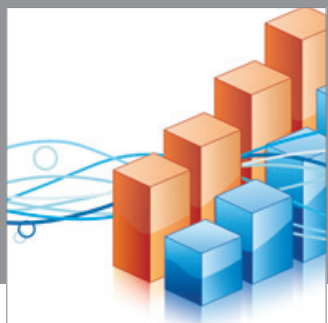

Advances in

Operations Research

mansans

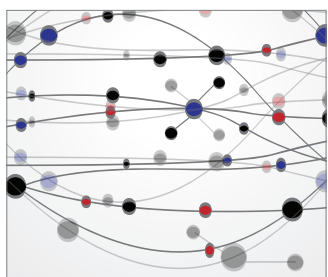

The Scientific World Journal
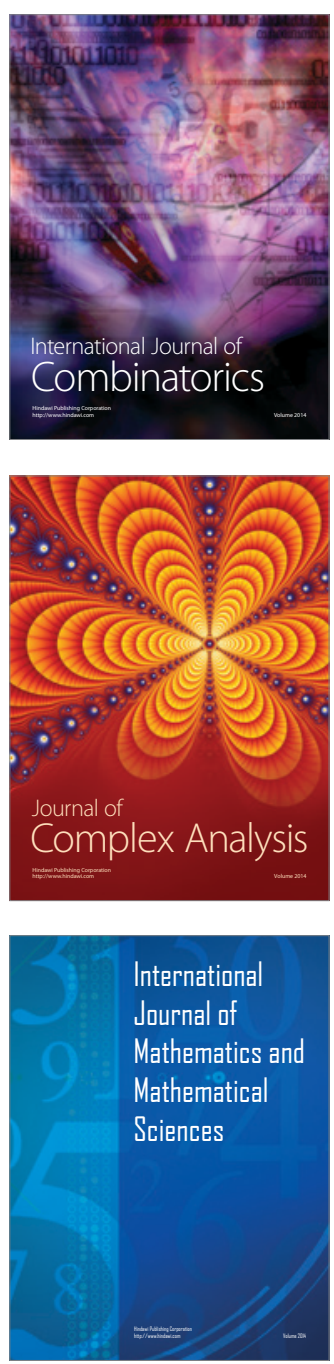
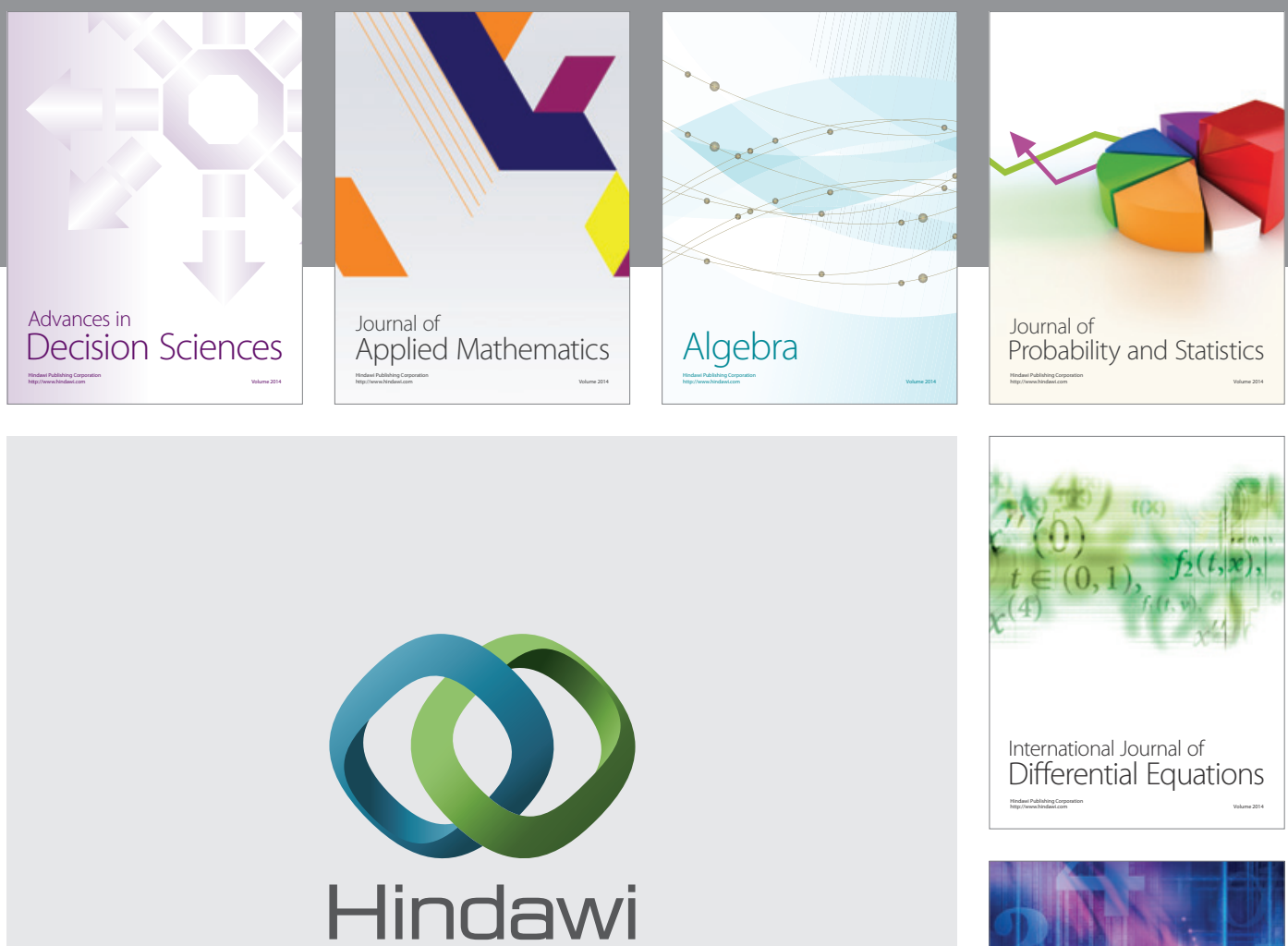

Submit your manuscripts at http://www.hindawi.com
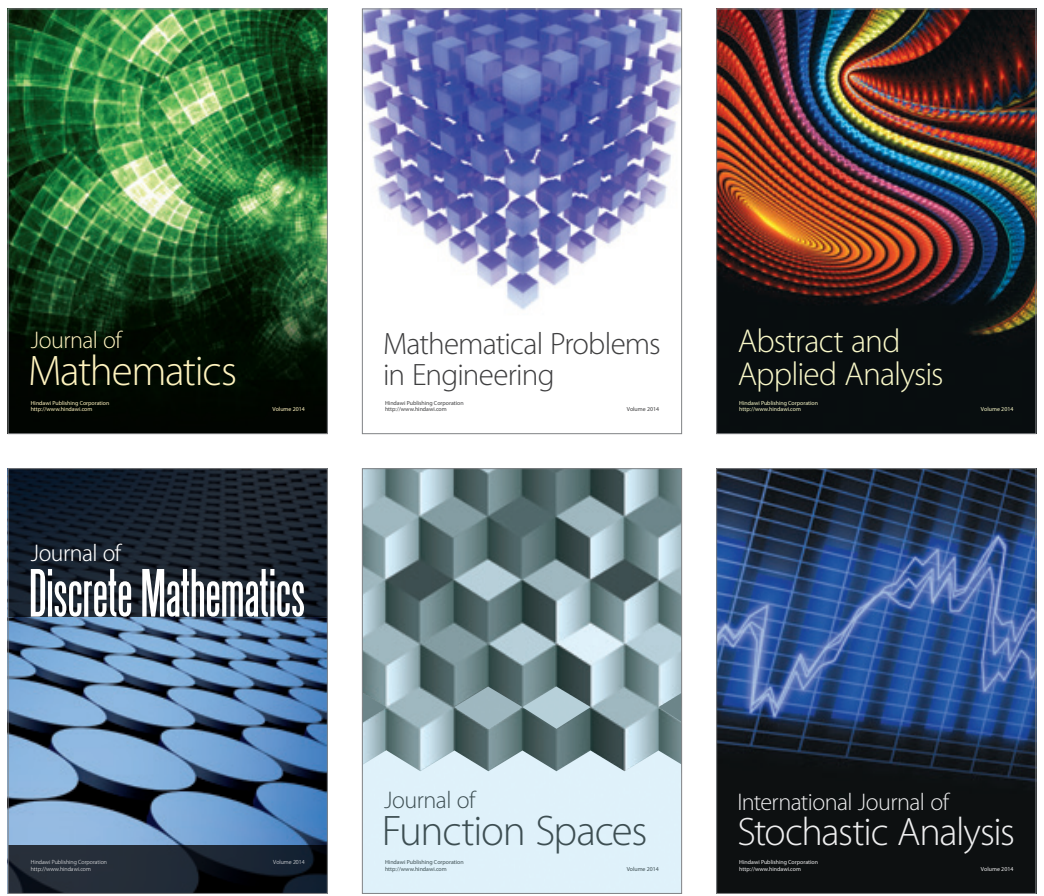

Journal of

Function Spaces

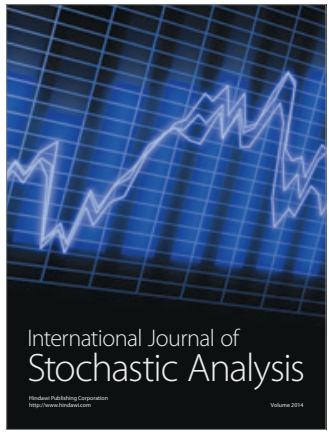

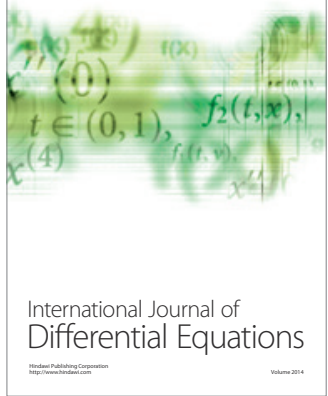
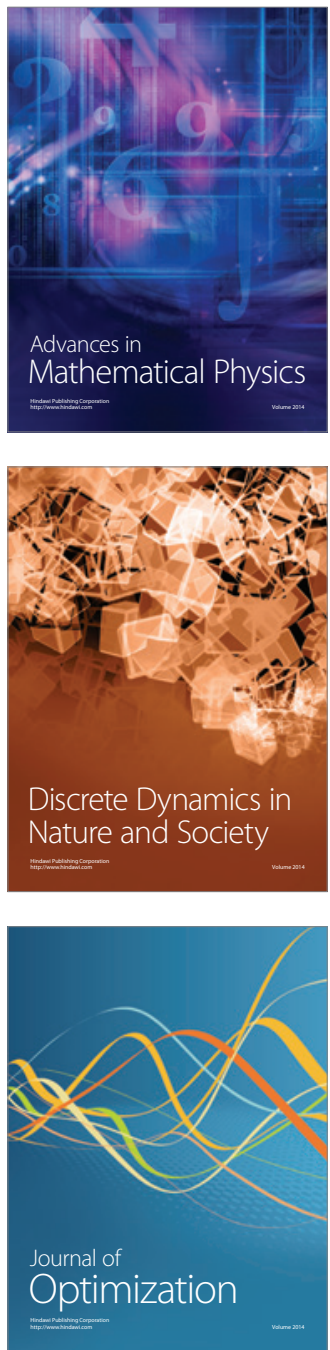